\title{
Analysis of Diagonal Horns Through Gaussian-Hermite Modes
}

\author{
Stafford Withington and J. Anthony Murphy
}

\begin{abstract}
The radiation characteristics of diagonal horns are investigated by means of Gaussian-Hermite modes. It is shown that, for reasonably long horns, the beamwidths in the principal and $45^{\circ}$ planes are equal to within $10 \%$, and all sidelobes are below $-15 \mathrm{~dB}$. It is also demonstrated that the phase center of a diffraction-limited horn is close to the aperture, whereas the phase center of a constant-beamwidth horn is behind the throat. The maximum coupling to the lowest order co-polar Gaussian mode is $84 \%$, and the total amount of power coupled into the cross-polarized lobes is $9.5 \%$. More significantly, the aperture efficiency of a Cassegrain antenna fed by a diagonal horn has a maximum value of $81 \%$, which compares with $87 \%$ for a corrugated horn. The maximum efficiency is achieved when the aperture of a diffraction-limited horn is placed at a confocal tertiary focus, although a secondary focus gives an aperture efficiency that is only $10 \%$ lower, suggesting that diagonal horns are suitable for focal-plane arrays.
\end{abstract}

\section{INTRODUCTION}

$\mathrm{T}$ HE rapid development of high-performance corrugated horns [1] has meant that the diagonal horn has received very little attention since it was first described by Love in 1962 [2]. There are situations, however, in which the difficulty or expense of manufacturing a corrugated horn precludes its use, and in these situations the diagonal horn is an attractive alternative. For example, at long millimeter wavelengths, suspended $E$-plane circuits are often manufactured by machining a rectangular waveguide into a split block. Obviously, corrugated and conical horns cannot be manufactured in this way, and even pyramidal horns, which have relatively poor radiation characteristics, are awkward to produce. A diagonal horn, on the other hand, can be milled into the block in a single operation and has good radiation characteristics; in fact, its beam efficiency in the $E$ - and $H$-planes is as good as that of a Potter horn [3] and is significantly better than that of a conical or pyramidal horn. In addition to being an inexpensive way of producing circularly symmetric beams at millimeter wavelengths, the diagonal horn is also attractive for submillimeter wavelengths where it becomes exceptionally difficult to electroform and interface conventional horns. In principle, it should be possible to manufacture compact arrays of diagonal horns for frequencies approaching $1 \mathrm{THz}$.

In his original paper, Love calculated the far-field radiation pattern of an infinitely long horn. That is to say he consid-

Manuscript received May 16, 1991; revised September 3, 1991.

$\mathrm{S}$. Withington is with Cavendish Laboratory, University of Cambridge. CB3 OHE, England.

J. A. Murphy is with Maynooth College, Kildare, Ireland. IEEE Log Number 9105954 ered, through diffraction integral techniques, the radiation characteristics of an open-ended diagonal waveguide. In this paper, we calculate the radiation characteristics of horns having finite lengths and therefore spherical phase fronts in their apertures. We analyze the horns by decomposing the aperture field into a number of Gauss-Hermite modes [4], each of which can be propagated in a simple manner into the Fresnel or Fraunhofer regions to reconstruct complex field patterns [5], [6]. Gaussian-mode analysis is, of course, a well-established technique for studying the behavior of longfocal-length millimeter- and submillimeter-wave systems [7]. It has the advantage over direct integration that one can propagate a diffracting beam through a complicated system of lenses, stops, and off-axis mirrors, and one can readily calculate the image that is formed across any surface within the system. In fact, in multimode Gaussian optics, diagonal horns of all lengths are characterized by one set of mode coefficients; the length of a given horn simply determines the amount by which the modes slip with respect to each other as the beam propagates. Love considered the radiation from a flat field in a diagonal aperture, and he therefore implied that the far-field phase center is in the plane of the aperture. Here, we calculate the position of the phase center directly by considering the phase perturbation caused by phase slippage between the modes.

The ability of a horn to produce low-order Gaussian modes is not in itself particularly interesting; usually one is more interested in the ability of a horn to illuminate, through suitable optics, a reflecting antenna. We therefore use the techniques of Lamb [8] and Padman [9] to study the aperture efficiency of a generic system comprising a diagonal horn, a quasi-optical beam-waveguide, and a Cassegrain antenna. A brief assessment of the horn's sensitivity to manufacturing tolerances is also made.

\section{Gaussian-Mode Analysis}

The aperture and Fraunhofer field distributions of a diffracted beam are related through a Fourier transform, and therefore the basis functions of a beam waveguide must be closely related to eigenfunctions of the Fourier transform. For noncircularly symmetric beams with tapering edges and reasonable focal lengths, the field can be expanded in terms of Gaussian-Hermite modes. Gaussian modes are only approximate solutions to the wave equation, but they are valid whenever the field propagates as a nearly plane wave. In practice, this restriction requires the focal length of the beam to be greater than about 2 as is usually the case for submillimeter-wave systems. When analyzing a horn through 
Gaussian modes, one essentially represents the radiated field in terms of the modes that would be radiated by a flat field in a virtual aperture. That is to say, the fields appear to be radiated by a virtual aperture that is some way behind the physical aperture, and the fields across the virtual aperture are, in contrast to those across the actual aperture, always flat. The problem is therefore one of determining the position and scale size of the virtual aperture and its effective field distribution.

In this paper, we consider a diagonal horn to be a section of gently tapering square waveguide supporting fully coherent spherically expanding $\mathrm{TE}_{10}$ and $\mathrm{TE}_{01}$ waveguide modes. We ignore mode conversion at the throat and along the length of the horn, and we assume that the modes are excited in phase. The field in the aperture of the horn has the basic form

$$
\mathbf{E}_{h}\left(x, y, z^{\prime}\right)=\mathbf{i} E_{01}+\mathbf{j} E_{10}
$$

or

$$
\begin{aligned}
\mathbf{E}_{h}\left(x, y, z^{\prime}\right)=\left(\mathbf{i} E_{x} \cos \frac{\pi y}{a}\right. & \left.+\mathbf{j} E_{y} \cos \frac{\pi x}{a}\right) \Pi\left(\frac{x}{a}, \frac{y}{z}\right) \\
\cdot \exp & {\left[-j \frac{\pi}{\lambda l}\left(x^{2}+y^{2}\right)\right] }
\end{aligned}
$$

where

$$
\Pi(x, y)= \begin{cases}1, & \text { if }|x| \text { and }|y| \leq \frac{1}{2} \\ 0, & \text { otherwise }\end{cases}
$$

and, as shown in Fig. 1, the coordinate frame is centered on the virtual aperture. The field has a form that is very similar to that of the $\mathrm{TE}_{11}$ mode of a conical horn; however, because the $E$-plane is more tapered one would expect the beam to be more nearly circular. In fact, the field around the edge of a diagonal aperture is zero on the principal planes and maximum on the $45^{\circ}$ planes, and therefore the longest axes have the most taper and the beam is nearly circular.

The field can also be expanded in terms of co-polar and cross-polar components, with respect to the diagonal, giving

$$
\mathbf{E}_{h}\left(x, y, z^{\prime}\right)=\mathbf{v} E^{+}+\mathbf{h} E^{-}
$$

where

$$
\begin{aligned}
& E^{ \pm}=\left(\frac{E_{x}}{\sqrt{2}} \cos \frac{\pi y}{a} \pm \frac{E_{y}}{\sqrt{2}}\right.\left.\cos \frac{\pi x}{a}\right) \Pi\left(\frac{x}{a}, \frac{y}{a}\right) \\
& \cdot \exp \left[-j \frac{\pi}{\lambda l}\left(x^{2}+y^{2}\right)\right]
\end{aligned}
$$

and the original coordinate frame has been retained because its boundary conditions are particularly simple. The field radiated by the diagonal horn can be written as a sum of free-space Gaussian-Hermite modes; more specifically,

$$
\begin{aligned}
\mathbf{E}_{h}(x, y, z)= & \frac{w\left(z^{\prime}\right)}{w(z)} \sum_{m} \sum_{n}\left(\mathbf{i} A_{m} B_{n}+\mathbf{j} B_{m} A_{n}\right) \\
& \cdot h_{m}\left(\frac{\sqrt{2} x}{w(z)}\right) h_{n}\left(\frac{\sqrt{2} y}{w(z)}\right)
\end{aligned}
$$
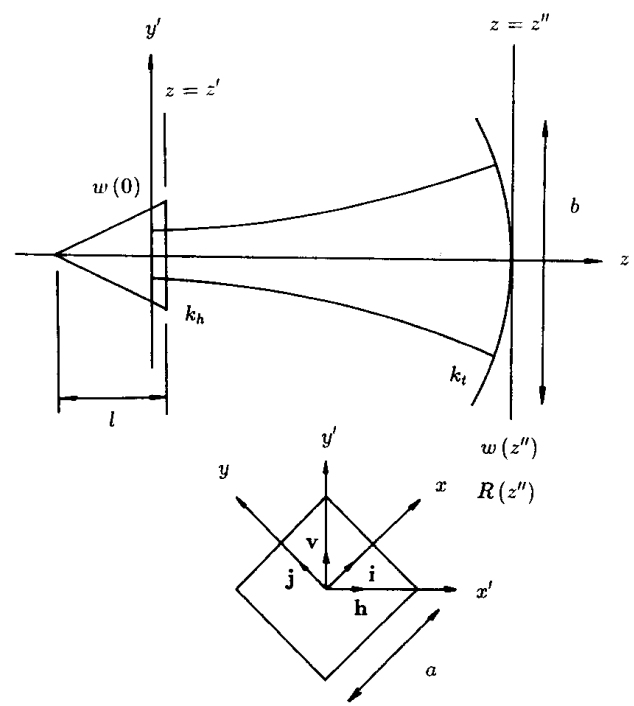

Fig. 1. The coordinate system used to analyze the radiation characteristics of diagonal horns.

$$
\begin{aligned}
& \cdot \exp \left[-j \frac{\pi}{\lambda R(z)}\left(x^{2}+y^{2}\right)\right] \\
& \cdot \exp \left[j(m+n+1) \tan ^{-1} \frac{z}{z_{c}}\right] \exp \left[j \phi_{m n}\right]
\end{aligned}
$$

where the dilution factor $w\left(z^{\prime}\right) / w(z)$ is referenced to the field in the aperture, and for the moment we have taken advantage of the rotational symmetry of the $\mathrm{TE}_{10}$ and $\mathrm{TE}_{01}$ fields by setting $E_{x}=E_{y}$. The basis functions

$$
h_{m}(u)=\frac{H_{m}(u) \exp \left[-\frac{u^{2}}{2}\right]}{\left(\sqrt{\pi} 2^{m} m !\right)^{1 / 2}}
$$

are orthonormal in the sense that

$$
\int_{-\infty}^{+\infty} h_{m}(u) h_{n}(u) d u=\delta_{m n}
$$

where $H_{m}(u)$ is the Hermite polynomial of order $m$ in $u$. The beam scale size and the equiphase radius of curvature are common to all modes, and they are given by

$$
\begin{gathered}
w(z)=w(0)\left[1+\left(\frac{z}{z_{c}}\right)^{2}\right]^{1 / 2} \text { and } \\
R(z)=z\left[1+\left(\frac{z_{c}}{z}\right)^{2}\right]
\end{gathered}
$$

respectively. The beam scale size $w(z)$ is simply the radial distance between the axis of propagation and the position at which the field of the lowest order Gaussian mode has fallen to $1 / e$ of its on-axis value. The beam scale size is a function of position and it has a minimum value of $w(0)$ at the beam 
waist. The radius of curvature at the beam waist is infinite and therefore the beam waist acts as a virtual aperture from which the radiated fields diffract. Also,

$$
z_{c} \triangleq \frac{\pi w(0)^{2}}{\lambda}
$$

is the confocal distance, which essentially marks the boundary between the near and far fields, $\tan ^{-1} z / z_{c}$ is the phase slippage per mode, and $\phi_{m n}$ is an arbitrary mode-dependent phase constant. The phase slippage term is particularly interesting. As the name suggests, it describes the fact that a phase difference develops between modes as they propagate; in effect the modes "slip" with respect to each other. To the negative- $z$ side of a waist the phase front is retarded, whereas to the positive $z$ side of a waist the phase front is advanced. The total phase slippage as one moves through a waist is, according to the Gouy effect, $\pi$, and the maximum phase slippage between the waist and the far field is $\pi / 2$. It is straightforward to show that a phase slippage of $\pi / 2$ generates the angular Fourier transform of the field distribution at the waist. Now, as a beam propagates through a number quadratic focusing elements, such as thin lenses and long-focal-length off-axis mirrors, a phase slippage larger than $\pi / 2$ accumulates. The microscopic structure of the modified field is then determined by the total phase slippage, and the macroscopic structure is determined by the new beam waist and radius of curvature.

In Gaussian-Hermite modes and associated-Laguerre modes, as distinct from ordinary Laguerre modes, a phase slippage of $\pi$ corresponds to a real inverted image. This image may of course exist on a spherical surface as determined by the common radius of curvature. The inversion is easy to understand because two-dimensional transverse modes having $(m+n)$ odd have one-fold rotational symmetry and are negated, whereas modes having $(m+n)$ even have, in general, two-fold rotational symmetry and are unchanged. The net effect is to rotate the image by $\pi$. It should be noted that although the field in the aperture of a diagonal horn is not circularly symmetric it is invariant to rotation by $\pi$, and therefore phase slippages of 0 and $\pi$ are equivalent.

It is convenient to perform the overlap integral over an equiphase surface at the aperture, giving

$$
A_{m}=\left(\frac{k_{h}}{\sqrt{2}}\right)^{1 / 2} \int_{-1 / \sqrt{2} k_{h}}^{1 / \sqrt{2} k_{h}} h_{m}(u) d u
$$

and

$$
B_{n}=\left(\sqrt{2} k_{h}\right)^{1 / 2} \int_{-1 / \sqrt{2} k_{h}}^{1 / \sqrt{2} k_{h}} \cos \left(\frac{\pi k_{h} u}{\sqrt{2}}\right) h_{n}(u) d u
$$

where $k_{h} \triangleq w\left(z^{\prime}\right) / a$. The $x$ and $y$ dependencies have been factored, and the coefficients have been normalized to make the total generalized power in each waveguide mode unity:

$$
\sum_{m} \sum_{n}\left(A_{m} B_{n}\right)^{2}=1
$$

Furthermore, the mode coefficients are made real by choosing

$$
R\left(z^{\prime}\right)=l \text { and } \phi_{m n}=-(m+n+1) \tan ^{-1} \frac{z^{\prime}}{z_{c}} .
$$

There is a complete set of orthonormal modes associated with each value of $k_{h}$, and therefore we can choose $k_{h}$ in any manner we wish. For example, it can be chosen to optimize the power in the lowest order mode [6], it can be chosen to optimize the total amount of power contained in finite number of modes [5], or it can be chosen to minimize the contribution of phase slippage to the far-field radius of curvature [10]. The coupling of the lowest order one-dimensional Gaussian mode to a truncated uniform field has its maximum value when $k_{h}=0.51$ and to a cosine field when $k_{h}=0.35$. The coupling of the lowest order two-dimensional Gaussian mode, with the beam waists in each of the coordinates equal, to a square $\mathrm{TE}_{10}$ waveguide field has its maximum value when $k_{h}=0.43$. In the latter case, $84 \%$ of the power is contained in the beam, which compares with $98 \%$ for the $\mathrm{HE}_{11}$ mode of a corrugated horn.

As mentioned in the introduction, the coupling of a horn to the lowest order Gaussian mode is not in itself particularly interesting; usually, one is more interested in how efficiently a horn can be coupled to the diffraction-limited forward-lobe of a reflecting antenna, and this calculation requires higher order modes to be considered. For example, when $k_{h}=$ $0.43,97.9 \%$ of the power is contained in the first 25 nonzero modes, and $99.7 \%$ of the power is contained in the first 1000 modes. This slow increase in efficiency is due mainly to the difficulty of representing the sharp edges of the top-hat field distribution. However, if $k_{h}$ is reduced below its optimum value to 0.25 , then $98.8 \%$ of the power is contained in the first 25 modes, and $99.7 \%$ of the power is contained in the first 500 modes.

It should be emphasized that the mode coefficients do not depend upon the dimensions of the horn. The far-field power pattern of an individual horn is distinguished by the phase slippage between the modes at the aperture. For a narrowband or diffraction-limited horn, the physical aperture is in the near field of the virtual aperture, whereas for a wide-band or constant-beamwidth horn [11], the physical aperture is in the far field of the virtual aperture, or equivalently the waist of the mode set being used.

Once $k_{h}$ and $R\left(z^{\prime}\right)$ are fixed, the position and scale size of the virtual aperture are also fixed through

and

$$
\begin{gathered}
\frac{z^{\prime}}{l}=\frac{1}{1+\left(\frac{1}{4 k_{h}^{2} 2 \pi s}\right)^{2}} \\
w(0)=\frac{k_{h} a}{\left[1+\left(4 k_{h}^{2} 2 \pi s\right)^{2}\right]^{1 / 2}}
\end{gathered}
$$

respectively, where $2 \pi s$ is the total phase delay across the aperture, or

$$
s \triangleq \frac{a^{2}}{8 \lambda l}
$$


Also note that the phase constant becomes

$$
\phi_{m n}=-(m+n+1) \tan ^{-1} 4 k_{h}^{2} 2 \pi s .
$$

The aperture field can now be written as

$$
\begin{aligned}
\mathbf{E}_{h}(x, y, z)= & \frac{w\left(z^{\prime}\right)}{w(z)} \sum_{m} \sum_{n}\left(\mathbf{v} C_{m n}^{+}+\mathbf{h} C_{m n}^{-}\right) \\
& \cdot h_{m}\left(\frac{\sqrt{2} x}{w(z)}\right) h_{n}\left(\frac{\sqrt{2} y}{w(z)}\right) \\
& \cdot \exp \left[-j \frac{\pi}{\lambda R(z)}\left(x^{2}+y^{2}\right)\right] \\
& \cdot \exp \left[j(m+n+1) \tan ^{-1} \frac{z}{z_{c}}\right] \exp \left[j \phi_{m n}\right]
\end{aligned}
$$

where the co-polar and cross-polar mode coefficients are given by

$$
C_{m n}^{ \pm}=\frac{\sqrt{\Omega} A_{m} B_{n} \pm B_{m} A_{n}}{\sqrt{2(\Omega+1)}}
$$

and we have introduced the power balance factor

$$
\Omega \triangleq\left(\frac{E_{x}}{E_{y}}\right)^{2}
$$

The co-polar and cross-polar mode coefficients have been normalized to make the total generalized power unity

$$
\sum_{m} \sum_{n}\left(C_{m n}^{+}\right)^{2}+\left(C_{m n}^{-}\right)^{2}=1
$$

\section{FAR-Field Radiation Patterns}

The power-density-expressed in directional coordinates in the original coordinate frame-across a far-field surface is given by

$$
\begin{aligned}
P^{ \pm}(x, y, z)= & \frac{w\left(z^{\prime}\right)^{2}}{w(z)^{2}} \mid \sum_{m} \sum_{n} C_{m n}^{ \pm} h_{m}\left(\frac{\sqrt{2} \pi w(0)}{a} \frac{a}{\lambda} \frac{x}{z}\right) \\
& h_{n}\left(\frac{\sqrt{2} \pi w(0)}{a} \frac{a}{\lambda} \frac{y}{z}\right) \\
& \left.\cdot \exp \left[j(m+n)\left(\frac{\pi}{2}-\tan ^{-1} 4 k_{h}^{2} 2 \pi s\right)\right]\right|^{2} .
\end{aligned}
$$

Usually one is only interested in the power density relative to the forward direction, and therefore the dilution factor can be ignored. The $E$ - and $H$-plane co-polar power patterns of various diagonal horns are shown in Fig. 2. The power patterns in the principal planes are equal and they have no cross-polar component. Similarly, the co-polar and crosspolar power patterns of the $45^{\circ}$ planes are shown in Fig. 3 . For reasonably long horns, the beamwidths at $-15 \mathrm{db}$ in the principal and $45^{\circ}$ planes are equal to within $10 \%$, and all

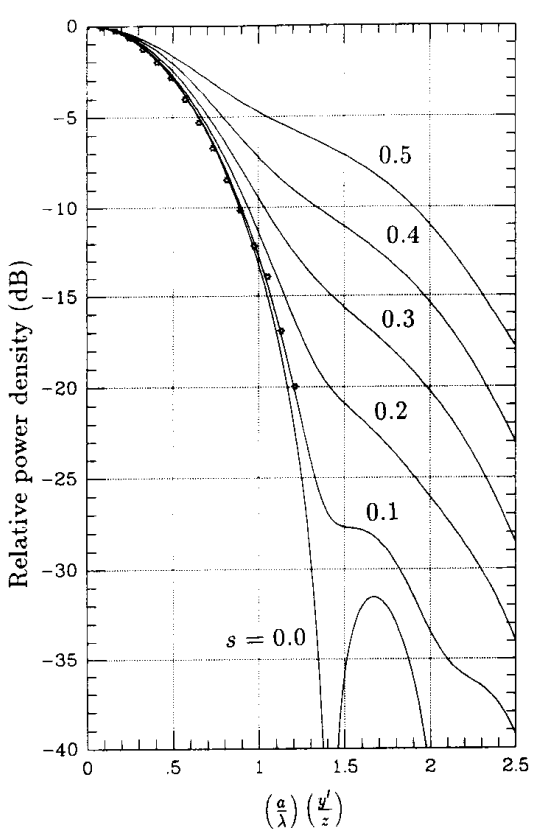

Fig. 2. The co-polar radiation pattern of a diagonal horn in the $0^{\circ}$ and $90^{\circ}$ planes for parametric values of the phase delay $2 \pi s$ across the aperture. The points show the measured characteristic of the submillimeter-wave horn described in the text; in this case, $s=0.16$.

sidelobes are below $-15 \mathrm{~dB}$. By contrast, a pyramidal horn has an $H$-plane half-power beamwidth that is $35 \%$ wider than the $E$-plane beamwidth. In Fig. 4, we show the effects of changing the power balance factor $\Omega$ to 0.6 . The diagram gives an indication of the horn's sensitivity to misalignments between the throat and the exciting rectangular waveguide. It can be seen that even when the power ratio between the waveguide modes is as high as 0.6 , the radiation patterns are almost unchanged. The main effect is to induce a central cross-polarized lobe of around $-18 \mathrm{~dB}$.

On Fig. 2 we have plotted the measured $E$-plane radiation pattern of a $345 \mathrm{GHz}$ diagonal horn having a sidelength of $4.5 \mathrm{~mm}$ and a length of $18 \mathrm{~mm}$. The horn was manufactured from tellurium copper by first routing $350 \times 700 \mu \mathrm{m}$ waveguide into each half of a split block. We found that it is possible to make an excellent quality waveguide by grinding a shaping tool with clearance angles of around $1.5^{\circ}$. The horn was machined by mounting the block at the flare angle of the horn $\tan ^{-1}(a / \sqrt{2} l)$ and tipping the head of the milling machine over at $45^{\circ}$. An ordinary end-mill can then be used to cut a V-shaped groove into the block. The rectangular to diagonal waveguide transition formed in this way is far from ideal, but we have found that for long horns the performance is not significantly affected by mode conversion. We have made horns for various frequencies and excellent quality and accuracy have been achieved. It should be possible to manufacture horns for very short submillimeter wavelengths by using this technique.

Another factor of interest when designing horns is the position of the phase center. In this paper, we understand the 


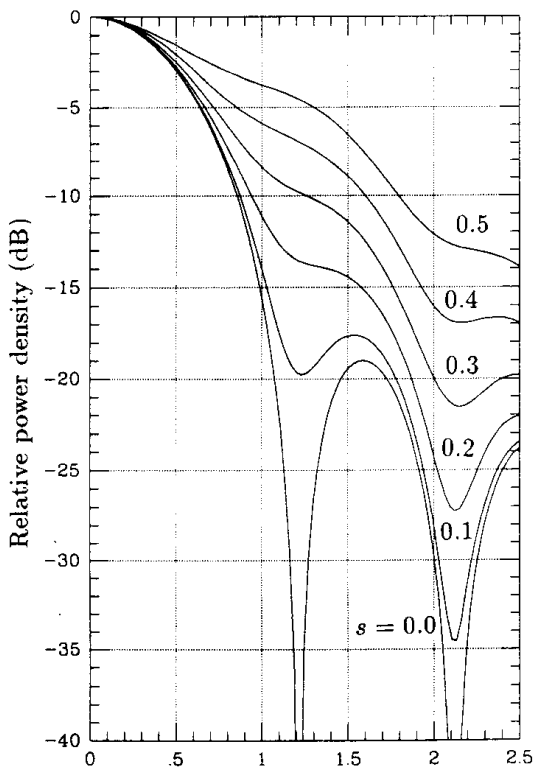

$\left(\frac{a}{\lambda}\right)\left(\frac{y}{2}\right)$

(a)

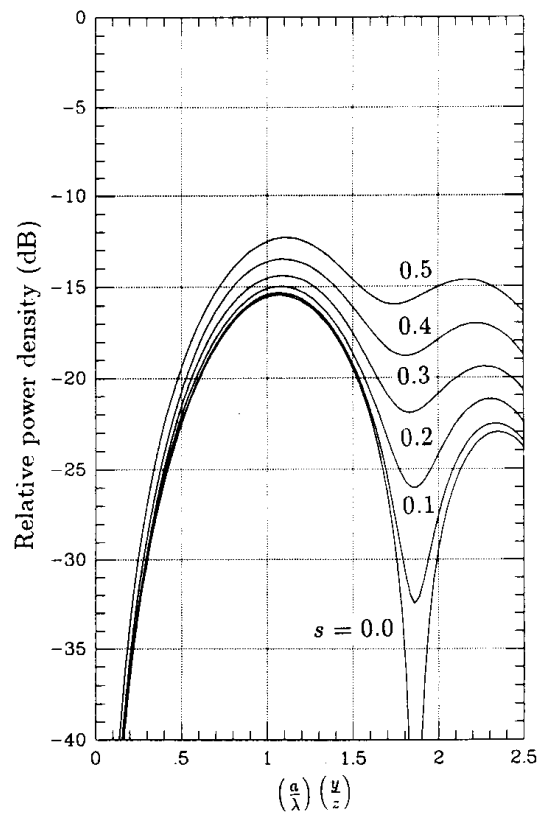

(b)

Fig. 3. The (a) co-polar and (b) cross-polar radiation patterns of a diagonal hom in the $45^{\circ}$ and $135^{\circ}$ planes for parametric values of the phase delay $2 \pi s$ across the aperture.

phase center to be the center of curvature of an equiphase surface on boresight. The position of the phase center depends, of course, on the distance of the measurement plane from the aperture. For example, the far-field phase center of a diffraction limited horn is at the aperture, whereas the aperture phase center is at the apex. It is important to

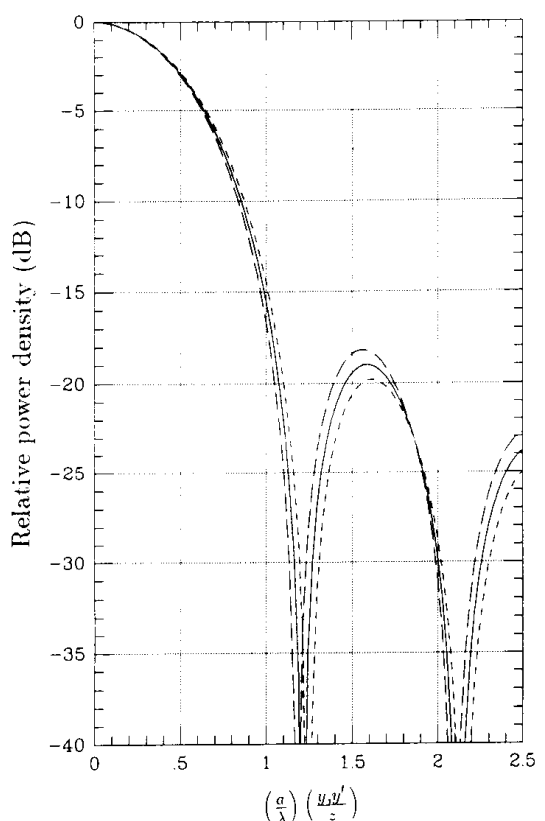

(a)

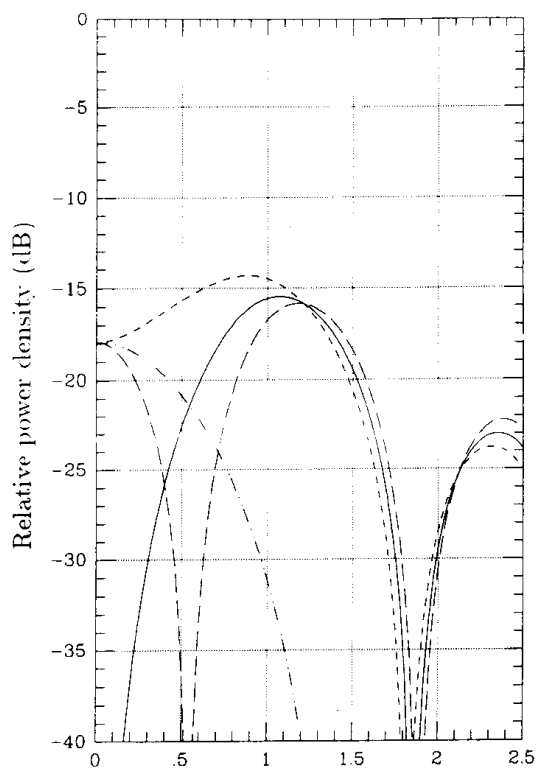

$\left(\frac{a}{\lambda}\right)\left(\frac{y, y^{\prime}}{z}\right)$

(b)

Fig. 4. The effect of $T E_{01}$ and $T E_{10}$ mode imbalance on the (a) co-polar and (b) cross-polar radiation patterns of a diffraction-limited diagonal horn. The various characteristics are as follows: The solid lines are for the $45^{\circ}$ and $135^{\circ}$ planes with the power balance factor $\Omega$ equal to unity; the short-dashed and long-dashed lines are for the $45^{\circ}$ and $135^{\circ}$ planes with $\Omega=0.6$; and the double-dot line is for the $0^{\circ}$ and $90^{\circ}$ planes with $\Omega=0.6$. The co-polar radiation patterns in the $0^{\circ}$ and $90^{\circ}$ planes are not affected by mode imbalance and are not shown. 
appreciate that in general there is only one value of $k_{h}$ for which the position of the far-field phase center is coincident with the position of the waist.

The position of the far-field phase center can be determined directly by expressing the phase of the radiated field in terms of a direction-dependent phase perturbation $\delta \theta$ superimposed on a distance-dependent spherical phase front:

$$
\begin{aligned}
& \angle \mathbf{E}_{h}(x, y, z)=\exp {\left[\delta \theta\left(\frac{x}{z}, \frac{y}{z}\right)\right] } \\
& \cdot \exp \left[-j \frac{\pi}{\lambda R(z)}\left(x^{2}+y^{2}\right)\right] .
\end{aligned}
$$

Consequently, the position of the phase center is determined by a combination of the phase introduced by mode slippage and that due to the common, or reference, radius of curvature. The perturbation is simply the phase error between two spherical surfaces and it indicates that the phase center is not at the same position as the waist. We can equate the above equation to an augmented quadratic phase term to get

$$
\delta \theta\left(\frac{x}{z}, \frac{y}{z}\right)=\left(\frac{\pi \delta z}{\lambda}\right)\left(\frac{r^{2}}{z^{2}}\right)
$$

where $\delta z$ is the distance between the far-field phase center and the position of the waist, and $r$ is the transverse distance $r^{2}=x^{2}+y^{2}$. The second derivative of the phase perturbation is thus a measure of sphericity, and the normalized distance between the phase center and the waist is given by

$$
\frac{\delta z}{l}=\frac{4 s}{\pi} \frac{\partial^{2} \delta \theta}{\partial\left(\frac{a}{\lambda} \frac{r}{z}\right)^{2}} .
$$

Fig. 5 shows the normalized distance between the principal-plane phase center and the aperture as a function of the phase delay across the aperture. For comparison, the position of the waist is also shown; however, the position of the waist depends on the value of $k_{h}$ chosen. In the present case we have chosen $k_{h}=0.43$ to maximize the coupling to the lowest order Gaussian mode. For low values of $s$ the horn is diffraction limited, and the waist and phase center are at the aperture; as $s$ increases the waist moves towards the vertex and the phase center moves behind the horn. The point at which the waist and phase center are coincident corresponds to the point at which mode slippage makes no contribution to the far-field phase. Also shown are a number of points carefully taken from the nomogram published by Muehldorf [12].

\section{Coupling EfFiciencies}

Before investigating aperture efficiency it is informative to consider the co-polar and cross-polar coupling efficiencies:

$$
\eta^{ \pm} \triangleq \frac{\int_{s}\left|E^{ \pm}\right|^{2} d s}{\int_{s}\left|E_{01}\right|^{2}+\left|E_{10}\right|^{2} d s}=\frac{1}{2} \pm \frac{8}{\pi^{2}} \frac{\sqrt{\Omega}}{(\Omega+1)} .
$$

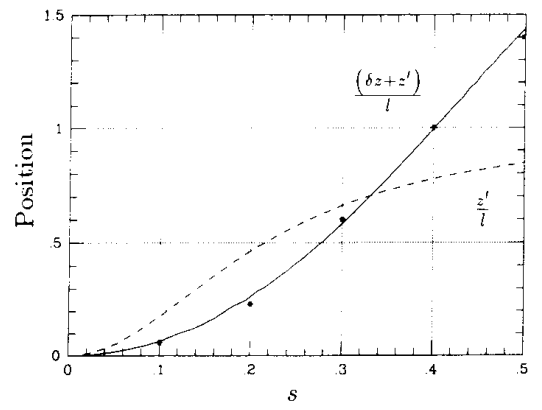

Fig. 5. The dotted line shows the normalized distance between the beam waist and the aperture, for $k_{h}=0.43$, as a function of the phase delay $2 \pi s$ across the aperture. The solid line shows the normalized distance between the phase center and the aperture as a function of the phase delay $2 \pi s$ across the aperture. The points are taken from the nomogram published by Muehldorf [11]

The efficiencies are shown as a function of the power balance factor in Fig. 6. Clearly, the amount of power coupled into the cross-polarized field is insensitive to mode imbalance. This characteristic further suggests that manufacturing tolerances are not critical, and that it should be possible to make good antennas at submillimeter wavelengths. The minimum amount of power coupled into the cross-polarized field is around $9.5 \%$, which is about the same as that of a conical horn.

Assessing the suitability of a horn for illuminating a Cassegrain antenna by measuring the "Gaussicity" of the far-field power pattern is clearly very unsatisfactory. Such an approach ignores structure in the phase of the fields-for example, it does not take into account focusing-and it also ignores power in higher order modes-the ideal horn has a top-hat field distribution in its aperture and therefore very high sidelobe levels. Instead, it is more meaningful to calculate the coupling integral between the field radiated by the horn and the field transmitted by the Cassegrain antenna illuminated by a point source at infinity. For a large, long-focal-length unblocked antenna, and ignoring diffraction by the subreflector, this problem is equivalent to considering the coupling of the horn to a top-hat field distribution of the form

$$
\mathbf{E}_{t}\left(x, y, z^{\prime \prime}\right)=\mathbf{v} E_{t} \Pi\left(\frac{r}{b}\right) \exp \left[-j \frac{\pi}{\lambda f} r^{2}\right]
$$

where $f$ is the focal length of an equivalent phase transforming surface located at $z^{\prime \prime}$. The aperture efficiency can be calculated by expanding the aperture field in terms of a set of Gaussian-Hermite modes similar to those used to expand the field associated with the horn. More specifically,

$$
\begin{aligned}
\mathbf{E}_{t}(x, y, z)= & \frac{w\left(z^{\prime \prime}\right)}{w(z)} \sum_{p} \sum_{q} T_{p q} h_{p}\left(\frac{\sqrt{2} x}{w(z)}\right) h_{q}\left(\frac{\sqrt{2} y}{w(z)}\right) \\
& \cdot \exp \left[-j \frac{\pi}{\lambda R(z)} r^{2}\right] \\
& \cdot \exp \left[j(p+q+1) \tan ^{-1} \frac{z}{z_{c}}\right] \exp \left[j \theta_{p q}\right] .
\end{aligned}
$$




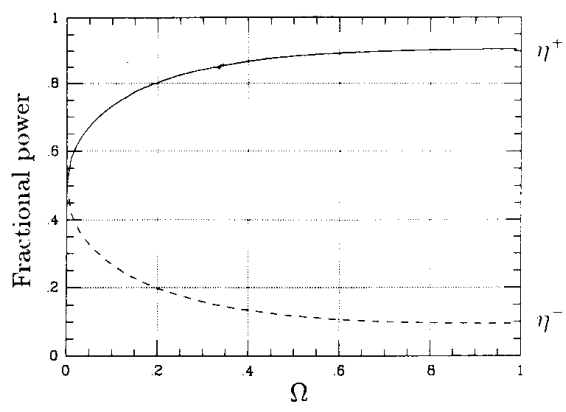

Fig. 6. The fractional amount of power coupled into the co-polar $\left(\eta^{+}\right)$and cross-polar $\left(\eta^{-}\right)$fields as a function of the $\mathrm{TE}_{01}$ and $\mathrm{TE}_{10}$ power balance factor $\Omega$.

To make the mode coefficients real one can choose

$$
R\left(z^{\prime \prime}\right)=f \text { and } \theta_{p q}=-(p+q+1) \tan ^{-1} \frac{z^{\prime \prime}}{z_{c}}
$$

and then

$$
T_{p q}=\left(\frac{2}{\pi}\right)^{2} k_{t} \int_{0}^{1 / \sqrt{2} k_{t}} \int_{0}^{2 \pi} h_{p}(u \cos \theta) h_{q}(u \sin \theta) u d u d \theta
$$

where $k_{t} \triangleq w\left(z^{\prime \prime}\right) / b$. Once again the mode coefficients have been normalized to make the total power unity

$$
\sum_{p q} T_{p q}^{2}=1
$$

With $k_{h}=0.43$, which is close to that required for maximizing the coupling to the lowest order mode, only $96 \%$ of the power is contained in the first 400 nonzero modes. A large number of modes are therefore required in order to calculate aperture efficiencies accurately. A large number of coherent modes does not, of course, necessarily imply that the field is distributed over a large region of space.

Defining the aperture efficiency as

$$
\eta \triangleq\left|\int_{s} \mathbf{E}_{h} \mathbf{E}_{t}^{*} d s\right|^{2}
$$

leads to

$$
\eta=\left|\sum_{m} \sum_{n} C_{m n}^{+} T_{m n} \exp \left[-j \delta_{m n}\right]\right|^{2}
$$

where the coupling integral has been evaluated over the aperture of the horn. We have set the waist of the horn modes and the waist of the telescope modes at the aperture of the horn equal:

$$
w_{h}\left(z^{\prime}\right)=w_{t}\left(z^{\prime}\right) \text {. }
$$

Furthermore, we have set the radius of curvature of the telescope modes at the aperture of the horn equal to the length of the horn:

$$
R_{t}\left(z^{\prime}\right)=l \text {. }
$$

The mode-dependent phase slippage between the antenna and the aperture of the horn is then

$$
\delta_{m n}=(m+n)\left[\tan ^{-1} \frac{z^{\prime}}{z_{c}}-\tan ^{-1} \frac{z^{\prime \prime}}{z_{c}}\right]
$$

where the confocal distance is that of the antenna.

Fig. 7 shows contour plots of the aperture efficiency of an ideal Cassegrain antenna, having zero blockage, as a function of the normalized aperture size $k_{h}$ and the phase slippage per mode between the horn aperture and the antenna aperture. The first three plots are for parametric values of the antenna size $\left(k_{t}\right)$, and the last plot is for a power balance factor of 0.6 . The aperture efficiency is greatest for a diffraction-limited horn with its aperture at a confocal tertiary focus, although a secondary focus gives an efficiency that is only $10 \%$ lower. The plots show that the maximum attainable efficiency is around $81 \%$, which compares favorably with $87 \%$ for a corrugated horn and $82 \%$ for a Gaussian beam. Although, strictly speaking, the value of $81 \%$ is correct only to within a few percent due to the power lost in the decomposition, it is straightforward to show analytically that the maximum aperture efficiency is given by $J_{1}^{2}(\pi / 2) 8 / \pi$ or $82 \%$, which is about the same as that of a conical horn. Considering the relatively high polarization loss, $9.5 \%$, the aperture efficiency compares favorably with that of a corrugated horn. The sharp edges of the aperture distribution therefore partly compensate for the power lost in the cross-polar coupling. It should be noted that shaped optics will be less effective at improving the aperture efficiency of a diagonal horn than that of a corrugated horn. Somewhat conveniently, the aperture efficiency of a diagonal horn is greatest when the diameter of the image is equal to the side length of the horn.

Let us now consider the above analysis in more detail. For an antenna with a given focal ratio $F$, the value of $k_{t}$ fixes the mode set in terms of which the antenna and horn fields are expanded. The waist size is then given by

$$
w(0)=\frac{\lambda F}{\pi k_{t}}
$$

where we have assumed that the antenna is in the far field of its focus. Once the size of the waist is fixed, the radius of curvature across any surface is also fixed, and because we require the length of the horn to be equal to the radius of curvature, a horn of a given length can only be placed in one of two positions (assuming that the length of the horn is greater than twice the confocal distance). As the length of the horn is increased, one solution tends to a diffraction-limited horn with its aperture at a confocal secondary focus, whereas the other solution tends to a horn with its aperture at a confocal tertiary focus. In short, as far as analysis is concerned, one is limited as to where one can place a horn of a given length. If, however, one can vary $k_{t}$, then one can decouple the length of the horn and its position relative to the antenna. Once the horn has been positioned, changing $k_{h}$ changes the antenna to horn size ratio. Notice that, for a diffraction-limited horn at a confocal tertiary focus, $k_{h}$ at maximum efficiency is proportional to $k_{t}$, whereas for a diffraction-limited horn at a secondary focus, they are in- 

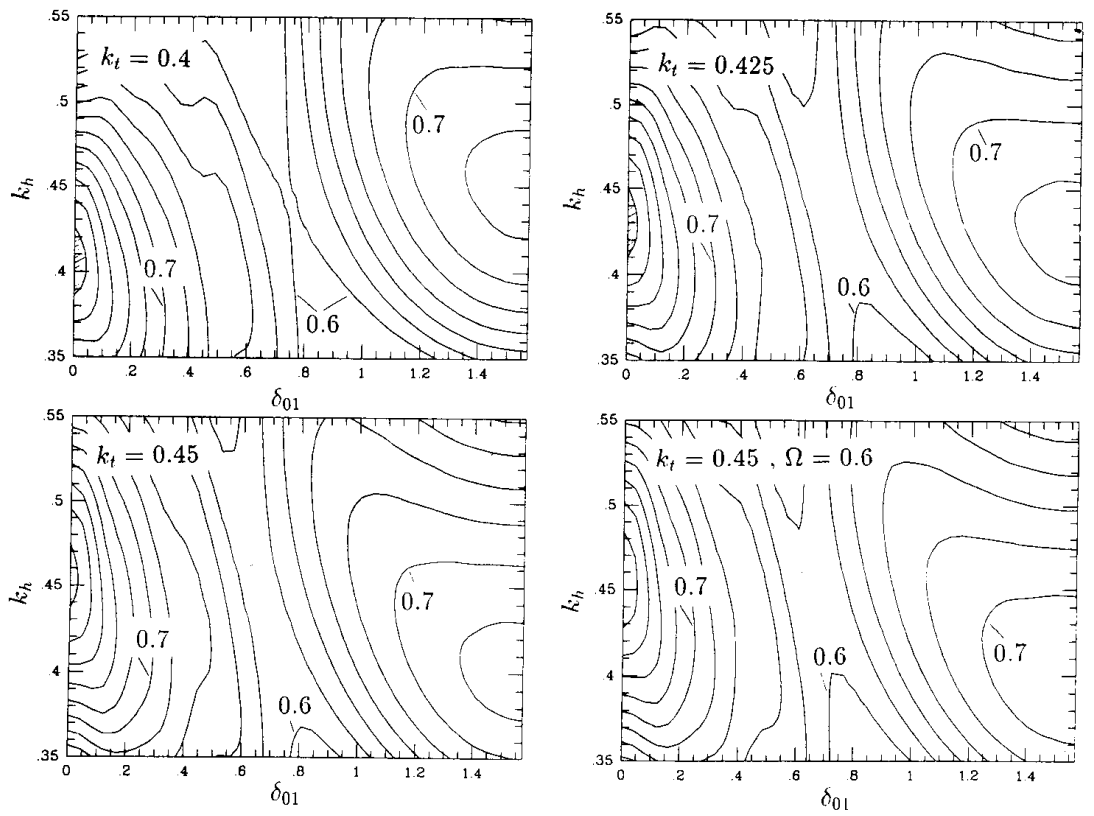

Fig. 7. The aperture efficiency of a Cassegrain antenna illuminated by a diagonal horn as a function of the horn-aperture size $k_{h}$ and the phase slippage per mode $\delta_{01}$ between the horn aperture and the antenna aperture. The first three plots are for parametric values of the antennas size $k_{t}$, and the last plot is for a $T E_{01}$ and $T E_{10}$ power balance factor $\Omega$ of 0.6 . The interval between the contour levels is 0.02 , and the regions of maximum aperture efficiency $\eta \geq 0.8$ are shaded.

versely proportional. This effect is a simple consequence of the Fourier relationship between the images.

The above analysis of aperture efficiency is somewhat artificial because it does not take into account the physical sizes of the components involved. In a real system, a Cassegrain antenna is usually combined with a number of focusing elements to make a theoretical design realizable. Nevertheless, the conclusions of the above analysis remain valid. If the focusing elements are confocal-that is to say, if the elements are separated by a distance equal to the sum of their focal lengths-then the images as determined by the accumulated phase slippage are plane. The image at a confocal secondary is a diffraction-limited image of the sky, or equivalently the Fourier transform of the aperture, whereas the image at a confocal tertiary is a scaled image of the aperture. In the latter case, the fields are frequency-independent and have limited spatial extent, and they are therefore ideal for coupling to the aperture of a diffraction-limited horn. In general, it is not easy to fabricate a horn having zero phase error across its aperture. One solution is to use a profiled design, but this approach is not appropriate for diagonal horns. An alternative solution is to recognize that if the focusing elements of the antenna system are nonconfocal, then the images, as determined by the phase slippage, are curved [13]. Indeed Padman [9] has shown that it is possible to use a single nonconfocal tertiary element to compensate for the phase error caused by the horn's finite length.

Finally, two practical comments are worthwhile. The diagonal horn achieves a respectable aperture efficiency by having sharp edges on its aperture distribution. Although a large number of modes are required in order to represent the distribution, the modes are coherent and they add to give frequency-independent top-hat type fields at tertiary focii and $\sin (x) / x$ type fields at secondary focii. Clearly, if there are unavoidable stops in a system, they should be positioned at tertiary focii. For example, if the horn is in a cryostat, then, ideally, two focusing elements should be placed in the cryostat to ensure that the window does not affect the aperture efficiency. A further issue is that diagonal horns have a $9.5 \%$ cross-polar coupling loss, and in a real system one would probably use a polarizing grid to terminate this loss in a well-defined way.

\section{CONCLUSION}

In this paper, we have used Gaussian-Hermite modes to study the radiation characteristics of finite-length diagonal horns. It has been shown that the coupling to a Gaussian beam is greatest when the waist-to-aperture size ratio is 0.43 , and that the optimum beam carries $84 \%$ of the total radiated power. The radiation pattern of a diagonal horn has almost perfect circular symmetry even when the amplitudes of the $\mathrm{TE}_{10}$ and $\mathrm{TE}_{01}$ waveguide modes are very different. For reasonably long horns, the beamwidths in the principal and $45^{\circ}$ planes are equal to within $10 \%$, and all sidelobes are below $-15 \mathrm{~dB}$. Mode imbalance leads mainly to the generation of a central cross-polarized lobe. We have presented a graph, calculated through considering mode slippage, showing the position of the far-field phase center as a function of the phase delay across the aperture. For long diffractionlimited horns, the phase center lies in the aperture, whereas for short constant-beamwidth horns, the phase center lies behind the throat. 
The coupling efficiency to the cross-polarized field has a minimum value of $9.5 \%$, and the maximum aperture efficiency of a system comprising a diagonal horn, a beam waveguide, and a Cassegrain antenna is $82 \%$, which compares with $87 \%$ for a corrugated horn. The aperture efficiency is greatest, and frequency independent, for a diffraction-limited horn with its aperture at a tertiary focus; a secondary focus gives an efficiency that is only $10 \%$ lower suggesting that diagonal horns are suitable for focal-plane imaging arrays.

In conclusion, diagonal horns offer an inexpensive way of producing circularly symmetric beams at millimeter and centimeter wavelengths. Their ease of manufacture and compatibility with $E$-plane circuits means that they are also attractive for submillimeter wavelengths where it is exceptionally difficult to manufacture and interface conventional horns.

\section{ACKNOWLEDGMENT}

The authors would like to thank $N$. Whyborn for bringing the diagonal horn to their attention and R. Padman and R. E. Hills for a number of important discussions.

\section{REFERENCES}

[1] P. J. B. Clarricoats and A. D. Olver, Corrugated Horns for Microwave Antennas. London, UK: Peregrinus, 1984.

[2] A. W. Love, "The diagonal horn antenna," Microwave J., vol. V. pp. $117-122,1962$

[3] P. D. Potter, "A new horn antenna with suppressed sidelobes and equal beamwidths," Microwave J., vol. XX, pp. 71-78, 1963.

[4] H. Kogelnik and T. Li, "Laser beams and resonators," Proc. IEEE, vol. 54, pp. 1312-1325, 1966.

[5] C. Aubry and D. Bitter, "Radiation pattern of a corrugated conical horn in terms of Laguerre-Gaussian functions," Electron. Lett. . vol. 11, pp. 154-156, 1975 .

[6] R. J. Wylde, "Millimetre-wave Gaussian beam-mode optics and corrugated fee horns," Proc. Inst. Elec. Eng., vol. 131, pp. $258-262,1984$.

[7] P. F. Goldsmith, 'Quasi-optical techniques at millimeter and submillimeter wavelengths," in Infrared and Millimeter Waves, vol. 6 . New York: Academic, 1982, pp. 277-343.

[8] J. W. Lamb, "Quasi-optical coupling of Gaussian beam systems to large Cassegrain antennas," Int. J. Infrared Millimeter Waves. vol. 7 , pp. $1511-1536,1986$.
[9] R. Padman, J. A. Murphy, and R. E. Hills, "Gaussian mode analysis of Cassegrain antenna efficiency." IEEE Trans. Antennas Propagat., vol. AP-35, pp. 1093-1103, 1987.

[10] J. A. Murphy and R. Padman. "Phase centers of horn antennas using Gaussian beam mode analysis," IEEE Trans. Antennas Propagat., vol. 38. pp. 1306-1310, 1990 .

$111]$ B. Mac. A. Thomas, "Design of corrugated conical horns," IEEE Trans. Antennas Propagat. vol. AP-26. pp. 367-372, 1978

[12] E. I. Muehldorf, "The phase center of horn antennas," IEEE Trans. Antennas Propagat.. vol. AP-18. pp. 753-760, 1970.

113] T. S. Chu, "An imaging beam waveguide feed," IEEE Trans. Antennas Propagat., vol. AP-31, pp. 614-619. 1983.

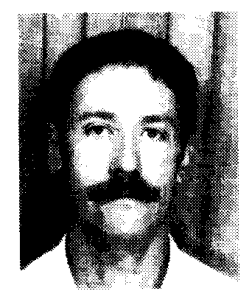

Stafford Withington was born in Chesterfield, England, on May 27. 1957. He received the B.Eng. Hons. degree, first class, from the University of Bradford, England, in 1979, and the Ph.D. degree in radio astronomy from the University of Manchester. England, in 1983.

He has held a number of positions, including a lectureship in the Department of Electrical Engineering at Sheffield University and a Royal Society Overseas Fellowship in the Department of Radio and Space Science at Chalmers University of Technology, Sweden. He is currently a Senior Research Associate in the Department of Physics at Cambridge University. He is also a College Lecturer at Downing College. Cambridge. In recent years he has concentrated on the development of low-noise submillimeter-wave receivers for use in radio astronomy, and he is particularly interested in superconducting detector technology and submillimeter wave optics.

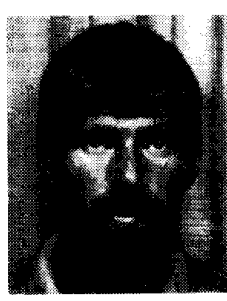

J. Anthony Murphy was born in Cork, Ireland, on October 30,1954. He received the B.Sc. and M.Sc, degrees in experimental physics from University College Cork. Ireland in 1977 and 1979. respectively, the M.S. degree in physics from the California Institute of Technology, Pasadena, in 1981, and the Ph.D. degree in Physics from the University of Cambridge, England in 1986.

From 1985 to 1986 he was a Research Associate at the Cavendish Laboratory where he was engaged in receiver development for the James Clerk Maxwell Telescope on Mauna Kea, Hawaii. In 1988 he was appointed as lecturer in the Experimental Physics Department, Maynooth College. Ireland. His main research interest is in millimeter- and submillimeter-wave optics. 